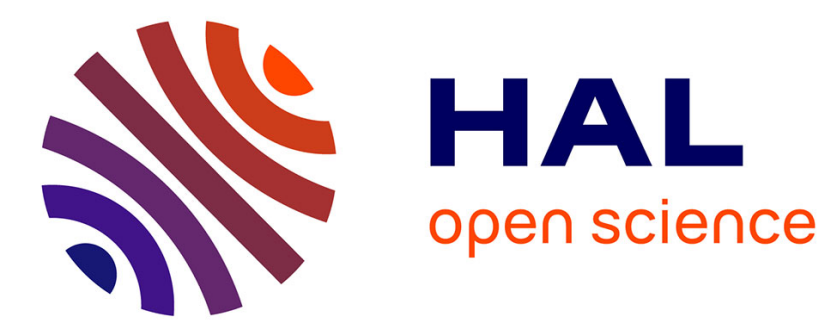

\title{
Dynamic Necking of Rods at High Strain Rates
}

\author{
V. Jeanclaude, C. Fressengeas
}

\section{To cite this version:}

V. Jeanclaude, C. Fressengeas. Dynamic Necking of Rods at High Strain Rates. Journal de Physique

IV Proceedings, 1997, 07 (C3), pp.C3-699-C3-704. 10.1051/jp4:19973119 . jpa-00255404

\section{HAL Id: jpa-00255404 https://hal.science/jpa-00255404}

Submitted on 1 Jan 1997

HAL is a multi-disciplinary open access archive for the deposit and dissemination of scientific research documents, whether they are published or not. The documents may come from teaching and research institutions in France or abroad, or from public or private research centers.
L'archive ouverte pluridisciplinaire HAL, est destinée au dépôt et à la diffusion de documents scientifiques de niveau recherche, publiés ou non, émanant des établissements d'enseignement et de recherche français ou étrangers, des laboratoires publics ou privés. 


\title{
Dynamic Necking of Rods at High Strain Rates
}

\author{
V. Jeanclaude and C. Fressengeas
}

L.P.M.M., U.R.A. $N^{\circ} 1215$ du C.N.R.S., Université de Metz, BP. 80794, Ile du Saulcy, 57012 Metz cedex 1, France

\begin{abstract}
The dynamic necking instability of a rod of a non-linear viscoplastic material as formed in shaped charges is investigated. A two-dimensional linear Lagrangian perturbation method leads to a single fourth order partial differential equation with time-dependent coefficients. The growth of disturbancies depends of the interplay between the stabilizing inertial and viscous effects, and the destabilizing geometrical softening of the rod. Inertia slows down the growth of long wavelengths, while viscosity damps preferentially the short wavelengths. A timeincreasing critical wavelength of maximum perturbation growth is selected at each moment. The latter is characteristic of the length scale of a multiple necking phenomenom.
\end{abstract}

\begin{abstract}
Résumé. On étudie la striction dynamique d'un jet (comportement viscoplastique non linéaire), obtenu par exemple à l'aide de charges creuses. Une méthode de perturbation linéaire Lagrangienne bi-dimensionnelle permet d'obtenir une équation aux dérivées partielles du quatrième ordre à coefficients dépendant du temps. La croissance des perturbations dépend d'une part des effets stabilisants de la viscosité et de l'inertie, et d'autre part des effets déstabilisants de l'adoucissement géométrique. L'inertie ralentit la croissance des grandes longueurs d'onde, alors que la viscosité influe plutôt sur les courtes longueurs d'onde. A chaque instant, il existe une longueur d'onde critique de croissance maximum des perturbations, caractérisant l'échelle de longueur d'un phénomène de striction multiple.
\end{abstract}

\section{INTRODUCTION}

High speed metallic jets are generated by the axisymmetric collapse of conical shells under explosive loading; during their flight, these jets experience large amounts of stretching at velocity gradients amounting to $10^{4}-10^{5} \mathrm{~s}^{-1}$. However, beyond a certain flight distance, they neck down in a series of locations, and break up into closely similar fragments. This particulation process is known to limit their perforating capabilities.

Here, the jet breakup is described as a dynamic necking phenomenon: a two dimensional dynamic necking instability analysis of a rod of a non linear viscoplastic material is carried out. Such a model can be traced back piecewise through the literature, but with assumptions which are not totally relevant herein: for a quasistatic deformation in Hutchinson and Obrecht [1], by neglecting the 2D features of the necking phenomenon in Chou and Carleone [2] or Yarin [3], for a Newtonian fluid in Frankel and Weihs [4], for perfectly plastic materials in Curtis [5] or Romero [6], and for a sheet in Fressengeas and Molinari [7].

In this paper, the influence of geometrical softening, inertial effects and of material rate-sensitivity on the necking morphology is investigated. It is believed that, although destabilizing thermal effects are likely to be significant, geometrical effects are responsible for the instability in the first place. Therefore, thermal effects, as well as deformation history prior to the jet formation, and material anisotropy are not considered. Surface tension and aerodynamic yield very small rates of growth of disturbancies [1]; like elastic waves, they are neglected. By using the perturbations relative amplification measure, the stabilizing effects of inertia and strain rate sensitivity on the growth of disturbances is evidenced. The plan of the paper is as follows: first, the exact non-linear Lagrangian formulation of the problem is set up and the basic flow is described. Next, the stability of the basic tension solution is investigated by using a Lagrangian linear perturbation analysis, and finally basic properties of the non uniform two-dimensional dynamic strain field are given.

\section{PROBLEM FORMULATION}

To describe the evolution of deformation instabilities that follow the overall material stretching, the Lagragian framework seems more suitable and will be used hereafter. It is convenient to use nondimensional variables: all length variables are scaled by the length $L$ of the specimen in the reference configuration, velocity variables are scaled by the constant velocity $V$ of the specimen tip relative to its rear 
end. The velocity gradients are scaled by the characteristic stretching rate $\mathrm{V} / \mathrm{L}$, and time by $\mathrm{L} / \mathrm{V}$. To scale stresses, the initial axial stress $\sigma^{0 *}$ of a rod stretching uniformly at the rate $\mathrm{V} / \mathrm{L}$ is used. All field quantities are considered to be functions of the Lagrangian cylindric coordinates $\underline{X}=\left(r_{0}, \theta_{0}, z_{0}\right)$, which serve as particle labels, and of the time t. Let $\underline{x}=(r, \theta, z)$ be the Eulerian coordinates, and $\underline{u}=\left(u_{r}, u_{\theta}, u_{z}\right)$ the velocity components. According to the symmetry of the rod, all quantities are assumed to be independant of $\theta_{0}$ and $u_{\theta}=0$. The rod is subjected to the velocity boundary conditions

$$
\mathrm{u}_{\mathrm{z}}=0 \quad \text { at } \mathrm{z}_{0}=0, \quad \mathrm{u}_{\mathrm{z}}=1 \quad \text { at } \mathrm{z}_{0}=1
$$

and to the symmetry condition

$$
\mathrm{u}_{\mathrm{r}}=0 \text { at } \mathrm{r}_{0}=0
$$

At any instant $t$ and position $z_{0}$, the outer surface is symmetrically disposed about the rod midline $r_{0}=0$, according to $r_{0}=\rho$ with $\rho=R_{a} / L\left(R_{a}\right.$ is the radius of the rod $)$. It is further assumed that the stress vector vanishes on the outer surface.

$$
\underline{t}=\underline{\sigma} \cdot \underline{v}=\underline{n} \cdot \underline{v}_{0}=0 \quad \text { at } \mathrm{r}_{0}=\rho
$$

$\underline{\sigma}$ and $\underline{\mathbf{n}}$ denote respectively the Cauchy stress tensor and the transpose of the nominal stress tensor (Boussinesq tensor); $\underline{v}$ and $\underline{v}_{0}$ are the outer surface normals in the present and reference configurations. In addition, it is required that the shear stress be zero on the rest of the boundaries

$$
\sigma_{\mathrm{rz}}=0 \quad \text { at } \mathrm{z}_{0}=0, \mathrm{z}_{0}=1, \quad \mathrm{r}_{0}=0
$$

The material is assumed to be incompressible

$$
\frac{\partial u_{r}}{\partial r}+\frac{u_{r}}{r}+\frac{\partial u_{z}}{\partial z}=0
$$

Using the Boussinesq tensor $\underline{\underline{n}}$, the Lagrangian momentum equations are written as

$$
\mathrm{R}_{0} \frac{\partial \underline{\mathrm{u}}}{\partial \mathrm{t}}=\operatorname{grad} \underline{\underline{n}}
$$

where $R_{0}=\rho_{0} \frac{V^{2}}{\sigma^{0^{*}}}$ is the Reynolds number ( $\rho_{0}$ is the mass density).

Let us denote by $\underline{\mathrm{D}}$ the strain rate tensor, by $\underline{\mathrm{s}}$ the Cauchy stress tensor deviator, and let us use in addition the equivalent stresss $\sigma_{\mathrm{e}}=\left(\frac{3 \mathrm{~s}: \underline{s}}{2}\right)^{1 / 2}$ and the equivalent strain rate $\mathrm{d}_{\mathrm{e}}=\left(\frac{2 \underline{\mathrm{D}}: \underline{\mathrm{D}}}{3}\right)^{1 / 2}$. The material strain rate sensitivity being $\mathrm{m}$, the viscoplastic behavior is specified by

$$
\underline{\mathrm{s}}=\Lambda \underline{\mathrm{D}}, \quad \Lambda=\frac{2 \sigma_{\mathrm{e}}}{3 \mathrm{~d}_{\mathrm{e}}}, \quad \sigma_{\mathrm{e}}=\mathrm{d}_{\mathrm{e}}^{\mathrm{m}}
$$

The relation $\sigma_{\mathrm{e}}^{*}=\mu \mathrm{d}_{\mathrm{e}}^{* \mathrm{~m}}$, with $\mu=\frac{\sigma^{0^{*}}}{(\mathrm{~V} / \mathrm{L})^{\mathrm{m}}}$, is the dimensional counterpart of (2-7-c).

It is straightforward to find the homogeneous solution of the equations $(2-1,2-7)$

$$
\left\{\begin{array}{ccc}
\mathrm{r}^{0}=\mathrm{r}_{0} \varepsilon^{1 / 2} & \mathrm{z}^{0}=\mathrm{z}_{0} \varepsilon^{\varepsilon^{-1}} \\
\mathrm{u}_{\mathrm{r}}^{0}=-\frac{\mathrm{r}_{0}}{2} \varepsilon^{\prime 3 / 2}=-\frac{\mathrm{r}^{0}}{2} \varepsilon^{\prime} & \mathrm{u}_{\mathrm{z}}^{0}=\mathrm{z}_{0}=\mathrm{z}^{0} \varepsilon^{\prime} \\
\sigma_{\mathrm{rr}}^{0}=\sigma_{\theta \theta}^{0}=-\mathrm{p}^{0} & \sigma_{\mathrm{rz}}^{0}=0 & \sigma_{\mathrm{zz}}^{0}=-\mathrm{p}^{0}+\sigma^{0}
\end{array}\right.
$$

where $\sigma^{0}=\varepsilon^{\prime} \mathrm{m}, \mathrm{p}^{0}=\frac{3}{8} \mathrm{R}_{0} \varepsilon^{\prime 3}\left(\rho^{2}-\mathrm{r}_{0}^{2}\right)$, with $\mathrm{d}_{\mathrm{e}}=\frac{1}{1+\mathrm{t}}=\varepsilon^{\prime}, \sigma_{\mathrm{e}}=\varepsilon^{\prime} \mathrm{m}$. The homogeneous associated stream function is: $\psi=\frac{\varepsilon^{\prime}}{2} \mathrm{r}_{0}{ }^{2} \mathrm{z}_{0}$.

In this paper, we consider situations where the inertial pressure $\mathrm{p}^{0}$ value amounts to the viscoplastic characteristic stress $\sigma^{0}$. In such a case, inertial and viscoplastic effects are of comparable magnitude. It is worth noting that these inertial effects do no stem from any transient loading of the rod, but from the unsteadiness of the uniform tensile loading itself. The stability of that unsteady fundamental solution is now investigated by using a linear perturbation analysis. 


\section{LINEAR PERTURBATION ANALYSIS}

Let us neglect higher order terms and search for pertubed solutions in the form

$$
\mathrm{f}=\mathrm{f}^{0}+\delta \mathrm{f}
$$

where $\mathrm{f}$ stands for every variable $\mathrm{r}, \mathrm{z}, \mathrm{u}_{\mathrm{r}}, \mathrm{u}_{\mathrm{z}}, \sigma_{\mathrm{ij}}, \mathrm{n}_{\mathrm{ij}}, \psi$. The substitution of these variables into the equations (2-1, 2-7), substraction of terms belonging to the fundamental solution (2-8) and retention of the first order terms lead to a set of linear differential equations. Using the linearized incompressibility relation, let us introduce the pertubed stream function $\delta \psi$ such as

$$
\left\{\begin{array}{c}
-\frac{1}{\mathrm{r}_{0}} \frac{\partial \delta \psi}{\partial \mathrm{z}_{0}}=\varepsilon^{\prime-1 / 2}\left(\delta \mathrm{u}_{\mathrm{r}}+\frac{\varepsilon^{\prime}}{2} \delta \mathrm{r}\right) \\
\frac{1}{\mathrm{r}_{0}} \frac{\partial \delta \psi}{\partial \mathrm{r}_{0}}=\varepsilon^{\prime}\left(\delta \mathrm{u}_{\mathrm{z}}-\varepsilon^{\prime} \delta \mathrm{z}\right)
\end{array}\right.
$$

Cross-differentiating the linearized momentum equations, and combining them in such a way that the pressure perturbation is eliminated, one obtains a single fourth-order partial differential equation with timedependent coefficients which governs the evolution of $\delta \psi$ :

$$
\begin{aligned}
3 R \varepsilon^{\prime 3}\left(\varepsilon^{\prime}-1 \frac{\partial O \delta \psi}{\partial \mathrm{t}}+\varepsilon^{\prime 2} \frac{\partial^{3} \delta \psi}{\partial \mathrm{z}_{0}^{2} \partial \mathrm{t}}\right. & \left.+20 \delta \psi-\varepsilon^{\prime} \frac{\partial^{2} \delta \psi}{\partial \mathrm{z}_{0}^{2}}\right) \\
= & \mathrm{O}^{2} \delta \psi-(1-3 \mathrm{~m}) \varepsilon^{3} \frac{\partial^{2} \mathrm{O} \delta \psi}{\partial \mathrm{z}_{0}^{2}}+\varepsilon^{\prime} \frac{\partial^{4} \delta \psi}{\partial \mathrm{z}_{0}^{4}}
\end{aligned}
$$

Here $\mathrm{R}=\mathrm{R}_{0} \varepsilon^{\prime-\mathrm{m}}$ and the differential operator $\mathrm{O}$ is defined by $\mathrm{O} \delta \psi=\frac{\partial^{2} \delta \psi}{\partial \mathrm{r}_{0}^{2}}-\frac{1}{\mathrm{r}_{0}} \frac{\partial \delta \psi}{\partial \mathrm{r}_{0}}$.

The linearization of the velocity boundary conditions (2-1) and their expression using the pertubed stream function lead to

$$
\delta \psi=0 \text { at } \mathrm{z}_{0}=0 \text {, and } \delta \psi=0 \text { at } \mathrm{z}_{0}=1
$$

which also enforces the zero shear stress condition (2-4) at the ends; similarly, the midplane symmetry conditions $(2-2,2-4)$ on the velocity and shear stress imply

$$
\delta \psi=0 \text { and } \frac{\partial}{\partial \mathrm{r}_{0}}\left(\frac{1}{\mathrm{r}_{0}} \frac{\partial \delta \psi}{\partial \mathrm{r}_{0}}\right)=0 \delta \psi=0 \text { at } \mathrm{r}_{0}=0
$$

From (2-3), the linearized stress-free boundary condition is

or, using the pertubed stream function

$$
\delta n_{\pi}=\delta n_{r \theta}=\delta n_{r z}=0 \text { at } r_{0}=\rho
$$

$\left\{\begin{array}{c}O \delta \psi-\varepsilon^{3} \frac{\partial^{2} \delta \psi}{\partial \mathrm{z}_{0}^{2}}=3 \rho \varepsilon^{\prime 7 / 2} \frac{\partial \delta \mathrm{r}}{\partial \mathrm{z}_{0}} \\ \varepsilon^{\prime-3} \frac{\partial \mathrm{O} \delta \psi}{\partial \mathrm{r}_{0}}-\frac{2}{\rho} \frac{\partial^{2} \delta \psi}{\partial \mathrm{z}_{0}^{2}}+3 \mathrm{~m} \frac{\partial^{3} \delta \psi}{\partial \mathrm{r}_{0} \partial \mathrm{z}_{0}^{2}}=3 \mathrm{R}\left(\varepsilon^{\prime-1} \frac{\partial^{2} \delta \psi}{\partial \mathrm{r}_{0} \partial \mathrm{t}}+2 \frac{\partial \delta \psi}{\partial \mathrm{r}_{0}}+\frac{3}{4} \rho^{2} \varepsilon^{\prime 7 / 2} \frac{\partial \delta \mathrm{r}}{\partial \mathrm{z}_{0}}\right)\end{array}\right.$ at $\mathrm{r}_{0}=\rho$

where, using (3-2), $\delta \mathrm{r}$ is given by

$$
\delta \mathrm{r}=\varepsilon^{\prime 1 / 2}\left(\delta \mathrm{r}_{0}-\frac{1}{\mathrm{r}_{0}} \int_{0}^{\mathrm{t}} \frac{\partial \delta \Psi}{\partial \mathrm{z}_{0}} \mathrm{~d} \tau\right)
$$

$\delta r_{0}$ denotes the initial value for $\delta$.

Although the problem has been stated in terms of the pertubed stream function, the main interest here is in the growth of the non-uniformity of the Eulerian radius $r=r\left(\rho, z_{0}, t\right)$. Let us use $a=\left(r-r^{0}\right) / r^{0}$ as a relative measure of the non-uniformity of the evolving radius (more meaningful when the change in the uniform radius $\mathrm{r}^{0}$ is large). Using (3-8), it is found that

$$
a=\frac{1}{\rho}\left(\delta \mathrm{r}_{0}-\frac{1}{\rho} \int_{0}^{\mathrm{t}} \frac{\partial \delta \psi}{\partial \mathrm{z}_{0}}\left(\rho, \mathrm{z}_{0}, \tau\right) \mathrm{d} \tau\right)
$$




\section{RESULTS AND DISCUSSION}

In the one-dimensional long wavelength quasi-static approximation, the instantaneous rate of growth

$$
a^{\prime} / a=\varepsilon^{\prime} / m
$$

is obtained from (3-8) and (3-9). Accordingly, all perturbations increase with time, and are uniformly damped by the material rate sensitivity. We shall consider the normalized relative rate of growth $\mathrm{G}=\mathrm{m} \mathrm{a}^{\prime} / \mathrm{a}$ in what follows. In the one-dimensional dynamic case, the long wavelength value of $\mathrm{G}$ is given, as a function of the Lagrangian wave number $\mathrm{k}_{0}$, as

$$
\mathrm{G}=\frac{m \varepsilon^{\prime}}{2 \mathrm{R}_{0}}\left[-\left(2 \mathrm{R}_{0}+\mathrm{mk}_{0}^{2}\right)+\left\{\left(2 \mathrm{R}_{0}+\mathrm{mk}_{0}^{2}\right)^{2}+4 \mathrm{R}_{0} \mathrm{k}_{0}^{2}\right\}^{1 / 2}\right]
$$

which retrieves the result given in [8] (see fig. 1). In the two-dimensional case, we now look for solutions of (3-3) in the form

$$
\delta \psi\left(\mathrm{r}_{0}, \mathrm{z}_{0}, \mathrm{t}\right)=\mathrm{r}_{0} \mathrm{~A}(\mathrm{t}) \sin \left(\mathrm{k}_{0} \mathrm{z}_{0}\right) \operatorname{Re}\left[\eta(\mathrm{t}) \mathrm{I}_{1}\left(\mathrm{l}_{0} \varepsilon^{3 / 2} \mathrm{r}_{0}\right)\right]
$$

where $\mathrm{k}_{0}$ (resp. $\mathrm{l}_{0}$ ) is the Lagrangian longitudinal (resp. radial) wave number of a perturbation which stretches with the material. $\mathrm{I}_{1}$ is the modified Bessel function of the first kind and order 1 ; $\operatorname{Re}[$.$] means the$ real part. This form allows to satisfy the boundary condition (3-6), but, in order to satisfy $(3-5), \mathrm{k}_{0}$ has to be a multiple of $\pi$. In the dynamic case, we have to resort to an approximation on the time derivative of (43 ): indeed the relation (4-1) suggests that, for m small enough, the rate of growth of the perturbations is much larger than the rate of change $\varepsilon^{\prime}$ of the uniform stretching, therefore we assume $\frac{\partial \delta \psi}{\partial \mathrm{t}}=\frac{\mathrm{A}^{\prime}(\mathrm{t})}{\mathrm{A}(\mathrm{t})} \delta \psi=\lambda \varepsilon^{\prime} \delta \psi$. That condition being fulfilled, the substitution of (4-3) into (3-3) leads to a fourth order algebraic equation for $\mathrm{l}_{0}$

$$
1_{0}^{4}+\left[(1-3 m) k_{0}^{2}-3 R(\lambda+2)\right] 1_{0}^{2}+k_{0}^{4}-3 R k_{0}^{2}(1-\lambda)=0
$$

The growth parameter $\lambda$ as well as four complex solutions for $l_{0}$ are found such that $\delta \psi$ satisfies (3-3) and the problem boundary conditions. In the quasi-static case $(R=0)$, the growth rate $G$ is obtained exactly in closed form. At the initial time $t=0$, this result reduces to that provided by Hutchinson and Obrecht [1] (see fig. 1), which it extends at later times. In the dynamic case, the dynamic growth rate $\mathrm{G}$ is provided with satisfying accuracy when $\mathrm{m}$ is small enough

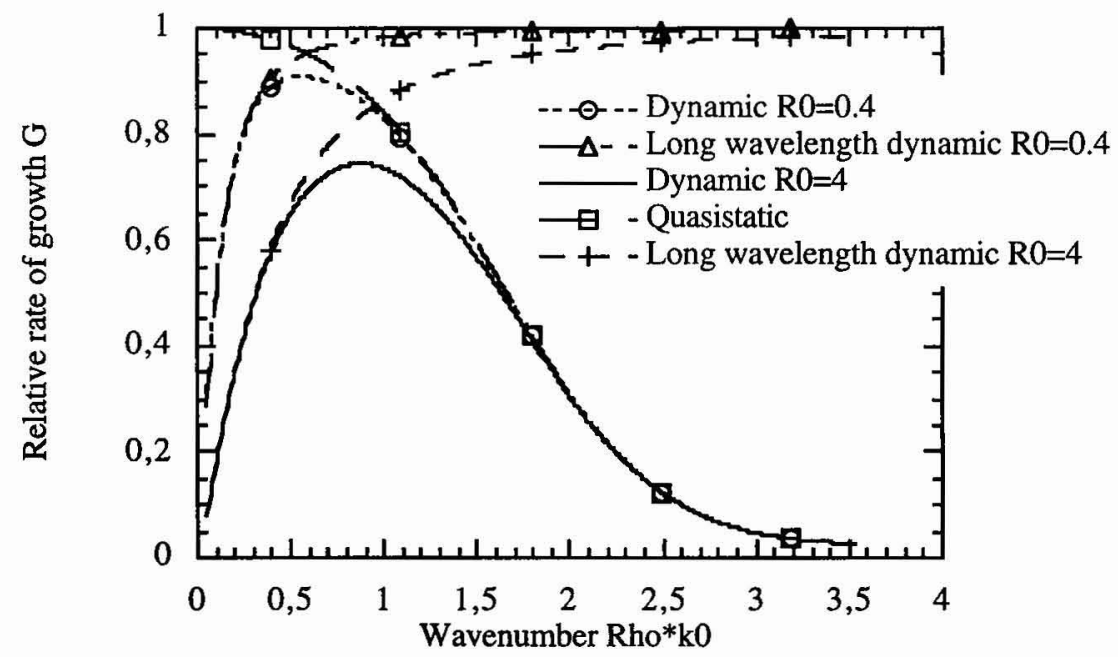

Figure 1: Initial dispersion curves $\left(m=0.05, R_{0}=0.4,4\right)$. Comparison with $1 D$ and quasi-static approximations. 
The dispersion curve $G$ vs. $k_{0} \rho$ is plotted in Figure 1 for $R_{0}=0.4,4$, and $m=0.05$, together with the one-dimensional dynamic approximation [8] and the two-dimensional quasi-static approximation [1] at time $t=0$. In the long wavelength limit, that is for $\mathrm{k}_{0} \rho<0.4$, G merges with the one-dimensional dynamic approximation (see eq. (4-2)), thus giving bounds for the validity of that simplier model. In the short wavelength limit (for $\mathrm{k}_{0} \rho>1.8$ ), G merges with the two-dimensional quasistatic approximation [1], and inertial effects can be neglected. At intermediate wavelengths $\left(0.4<\mathrm{k}_{0} \rho<1.8\right)$, a non-zero finite wavelength of maximum growth rate is selected. It is fundamental to note here that a maximum in the dispersion curves at a finite wavelength signals the occurrence of a multiple necking phenomenon, and the fragmentation of the rod at the outcome.

It can be seen also in Figure 1 that the larger the Reynolds number $R_{0}$, the wider the range of perturbations affected by inertial effects and the shorter the Lagrangian wavelength of maximum growth. In contrast, increasing the material rate sensitivity leads to longer critical wavelengths. This can be seen in Figure 2, where the initial dynamic dispersion curves are plotted for $R_{0}=4$, and for several rate-sensitivity values : $m=0.05,0.1,0.2$ and 0.5 . The larger the rate-sensitivity, the longer the critical wavelength of maximum growth, the smaller the range of significant inertial effects.

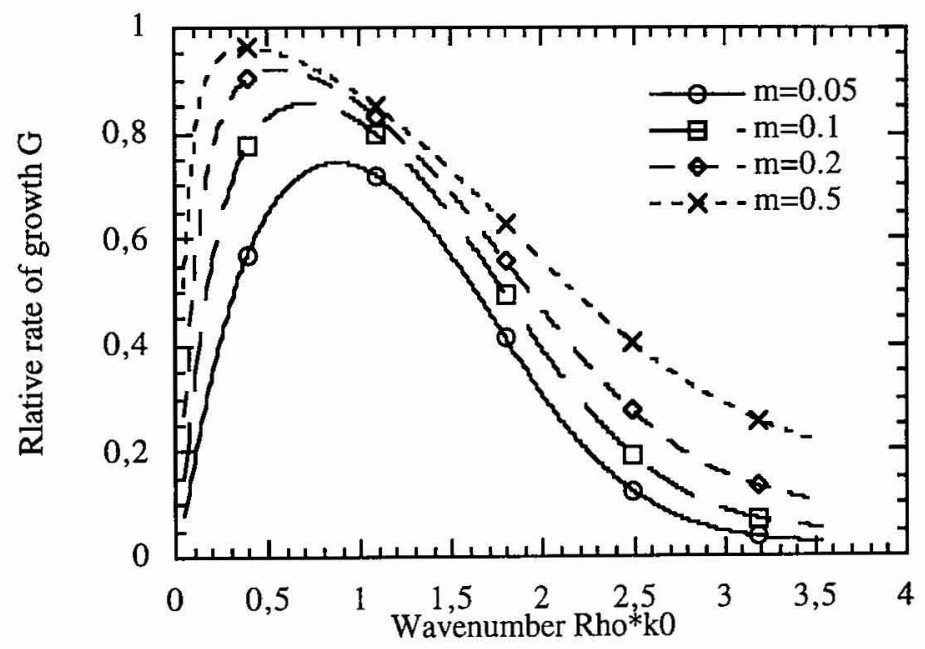

Figure 2: Influence of non-linear viscosity on the dynamic dispersion curve $\left(\mathrm{R}_{0}=4, \mathrm{t}=0\right) ; \mathrm{m}=0.05,0.1,0.2,0.5$.

It is shown in addition that he time evolution of the relative growth rate $\mathrm{G}$ is such that the dynamic Lagrangian wavenumber $\mathrm{k}_{\mathrm{m}}$ of the dominant perturbation is shifted to larger values as time goes on. Therefore, there is not a single dominant perturbation throughout the process, as in the quasistatic approximation, but rather a new one at each moment. The delayed modes will be in fine more significant of the observed instability pattern than the initially dominant modes.

\section{CONCLUSION}

In this paper, the dynamic necking instability of a uniformly stretching rod of a nonlinear viscoplastic material is investigated. A linear Lagrangian perturbation method accounting for inertial and twodimensional effects is used. The growth of disturbancies depends of the interplay between the stabilizing inertial and two-dimensional viscoplastic effects, and the destabilizing geometrical softening of the rod stemming from its section reduction. Inertia slows down the growth of long wavelengths, while twodimensional viscoplasticity damps preferentially the short wavelengths. Thus, at each moment, a dominant perturbation characterizes the structure of the velocity field. This critical wavelength is shifted with time towards larger values: therefore the initially dominant perturbations are not those who finally shape the fragmentation and provide the fragments size ratio. 
The model implies the following trends. Inertial and two-dimensional field effects are both stabilizing, since they delay the growth of all perturbations, compared to their quasi-static one-dimensional approximations. However, rods with higher rate-sensitivity, higher material strength or lower density yield larger critical wavelengths. Thus, if the material rate-sensitivity consistently favors the rod continuity, since fragments will come out later and longer, inertia plays a more ambiguous role by shortening the critical wavelength, as well as the fragments size at the outcome.

\section{References}

[1] Hutchinson J. W., and Obrecht H., 1977, Tensile instabilities in strain-rate dependent materials, Fracture 1977, ICF4 (Ed. D.M.R. TAPLIN), 1, 101, Waterloo-Canada.

[2] Chou P.C., Carleone J., 1977, The stability of shaped-charge jets, J. Appl. Phys., 48, 4187-4195.

[3] Yarin A.L., 1994, On instability of rapidly stretching metal jets produced by shaped charges, Int. J. Engng Sci. Vol. 32, No. 5, 847-862.

[4] Frankel I., Weihs D., 1985, Stability of a capillary jet with lenearly increasing axial velocity (with application to shaped charges), J. Fluid. Mech., 155, 289-307.

Frankel I., Weihs D., 1987, Influence of viscosity on the capillary instability of a stretching jet, J. Fluid. Mech., 185, 361-383.

[5] Curtis J.P., 1987, Axisymmetric instability model for shaped charge jets, J. Appl. Phys., 61, 49784985.

[6] Romero L.A., 1989, The instability of rapidly stretching plastic jets, J. Appl. Phys., 65, 3006-3016.

[7] Fressengeas C., Molinari A., 1994, Fragmentation of rapidly stretching sheets, Eur. J. Mech., 13, 251-268.

[8] Fressengeas C., Molinari A., 1985, Inertia and thermal effects on the localization of plastic flow, Acta. Met., 33, 387-396. 